\title{
Visible-light photoredox enables ketone carbonyl alkylation for easy access to tertiary alcohols
}

\author{
Minh Duy Vu, ${ }^{\dagger}$ Mrinmoy Das, ${ }^{\dagger}$ Aoxin Guo, Zi-En Ang, Miloš Đokić, Han Sen Soo and Xue-Wei Liu* \\ Division of Chemistry and Biological Chemistry, School of Physical and Mathematical Sciences Nanyang Technological \\ University 21 Nanyang Link, Singapore 637371
}

\begin{abstract}
Being a handle for synthesizing quaternary carbon centers and olefins, together with ubiquitous appearance in organic building blocks makes tertiary alcohols valuable targets in synthesis. However, traditional syntheses of these alcohols have faced several challenges including the employment of functionalized reactive reagents, undesirable side reactions and decomposition of the alcohol products under harsh conditions. The paucity of synthetic approach to bulky tertiary alcohols prompts our interest to develop a benign catalytic protocol to tackle the current issues. Here, we have successfully demonstrated the use of ketyl radicals in intermolecular cross radical-radical coupling, which has opened the new door for accessing complex tertiary alcohols. On the other hand, by starting from feedstock and naturally derived chemicals without any preactivation, it would be superior to traditional methodologies in industrial context.
\end{abstract}

\section{KEYWORDS photoredox catalysis, HAT catalyst, ketyl radical, tertiary alcohol, radical-radical cross coupling}

The tertiary alcohol scaffold is prevalent in organic molecules, ranging from pharmaceutical and agrochemical compounds to natural products. ${ }^{1}$ Traditional synthesis of alcohols, specifically tertiary alcohols, utilizes the Barbier type carbonyl addition reactions developed in the previous century. ${ }^{2}$ Nevertheless, the organometallic reagents involved are pyrophoric and highly reactive. As a consequence, they exhibit low functional group tolerance which limits their applicability in complex alcohol synthesis. Furthermore, the use of stoichiometric amounts of metallic reagents is unsustainable and harmful to the environment. Moreover, the nucleophilic addition of organometallic reagents to ketones is sometimes not as straightforward as compared to the reactions of aldehydes, owing to the inherent steric congestion around the carbonyl groups and the strong basicity of the reagents. Hence, these Barbier reactions are often accompanied by undesirable side reactions, including ketone reduction and aldol condensation, which lead to a complex mixture of side-products. ${ }^{3}$

In the last few years, a handful of new methodologies for carbonyl addition were invented to overcome such drawbacks. For example, milder organometallic reagents were developed to eliminate the anhydrous property of the reaction and many carbonyl additions can now be done in aqueous solvent. However, stoichiometric usage of metallic reagents is still necessary. ${ }^{4-6}$ These include nickel, copper and palladium, which catalyzes the direct reductive coupling between organic halides and carbonyl derivatives. ${ }^{7}$ Moving beyond the traditional design of carbonyl addition reactions, Krische presented the first reductive coupling between olefin-derived nucleophiles and primary/secondary alcohols. ${ }^{8}$ The concept of using unsaturated hydrocarbons as carbanion equivalent was also demonstrated by Buchwald and Liu through the discovery of copper-catalyzed

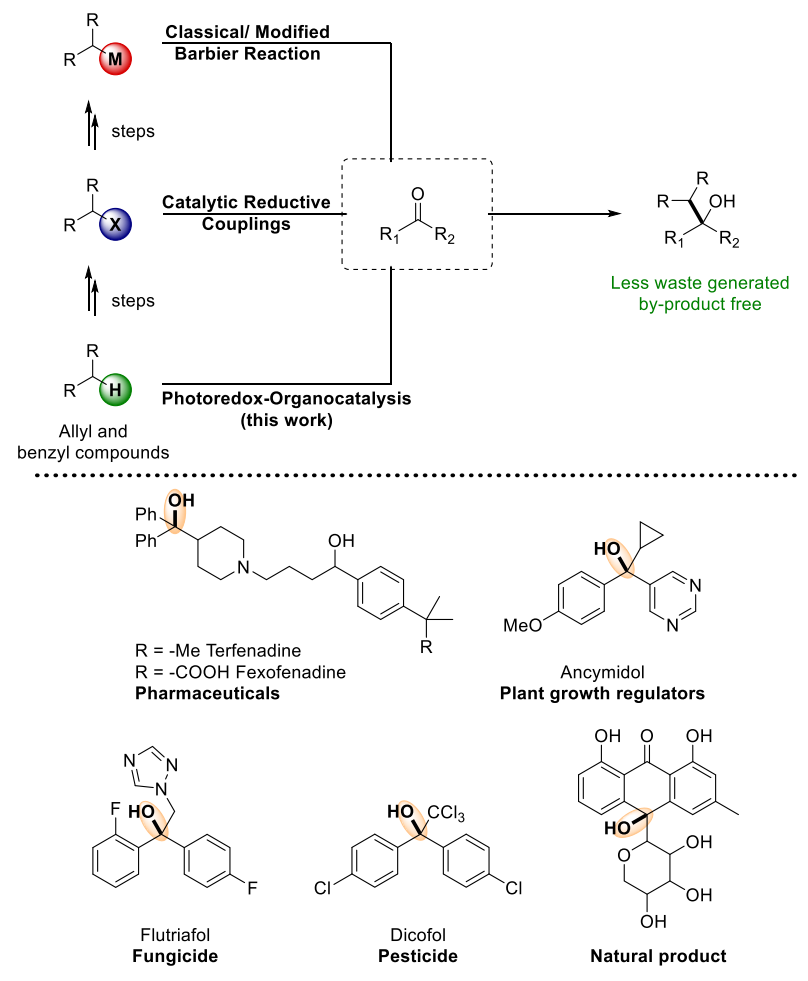

Figure 1. Overview of tertiary alcohol synthetic pathways and examples of its occurrence in natural and artificial products 1.

asymmetric addition to ketones. ${ }^{9}$ Subsequently, aldehydes were harnessed as latent alkyl carbanions for the ruthenium-catalyzed addition to carbonyl compounds, as reported by Li et al. ${ }^{10}$ The trend of reinventing the carbonyl addition reaction not only covered charge interaction mechanisms, but also extended to the field of radical chemistry. 
In 2016, Chi and co-workers demonstrated a metal-free process using carbene catalyst to facilitate the addition of benzyl halides to activated ketone substrates via a proposed radical pathway. ${ }^{11}$ Glorius discovered rare examples of intermolecular radical addition to carbonyl acceptors under photoredox conditions earlier this year. ${ }^{12}$ On the other hand, carbonyl substrates can be transformed into ketyl radical intermediates, which undergo subsequent $\mathrm{C}-\mathrm{C}$ bond formation, via samarium (II) halides - the potent single electron reductants. ${ }^{13-18}$

On the rise of photoredox chemistry over the last decade, the radical pathways that involve ketyl radicals have been developed extensively (Figure 2a). In 2013, Knowles and co-workers first reported the proton coupled electron transfer (PCET) approach to generate challenging ketyl radicals. These intermediates then underwent intramolecular cyclization with olefin or imine functional groups. ${ }^{19-20}$ The formation of ketyl radicals via photoredox catalysis was developed independently by Rueping through photoinduced electron transfer. ${ }^{21-23}$ It was revealed that under in situ activation of a hole catalyst such as an ammonium cation or an acid, the carbonyl reduction potential significantly drops so that a wide array of photoredox catalysts can be employed to achieve the ketyl radicals. Under the reaction condition, these radicals were found to dimerize ${ }^{22}$, couple with $\alpha$ amino radicals $^{21}$ and add to olefins ${ }^{23}$. In 2016, Ngai et al. reported another utility of ketyl radical - the intermolecular addition to electron deficient styrene derivatives. ${ }^{24-25}$

In comparison to olefin addition, cross radical-radical coupling of ketyl radicals may show broader applications. However, they are rarely reported in literature. The previous success on intramolecular version was presented by Zhu in 2016 in an attempt to synthesize multi-substituted $\mathrm{N}$-heterocycles. ${ }^{26}$ Another scarce example of intermolecular cross radical coupling between $\alpha$-heteroatom $(0, N)$ stabilized radicals and the ketyl radicals was developed by Xiao and co-workers. ${ }^{27-28}$ An asymmetric version of radical-radical cross coupling between electron deficient ketone and $\mathrm{N}$ methyl aryl amines was presented by Meggers and coworkers. ${ }^{29}$ Ooi et al. reported stereoselective cross coupling of $\mathrm{N}$ arylaminomethanes and aldimines using ionic Bronsted acid and photosensitizer. ${ }^{30}$ Earlier this year, König also developed photocatalytic Barbier reaction for allylation and benzylation of carbonyls. ${ }^{31}$ To the best of our knowledge, there has been no report on the use of simple transient radicals derived from hydrocarbon feedstock or natural sources in the analogous reaction.

In recent years, $\mathrm{C}-\mathrm{H}$ activation is a promising and highly favorable process for synthesis because of minimal prefunctionalization required on the substrates. ${ }^{32-34}$ Being intrigued by elegant approaches to transient radicals demonstrated by MacMillan in various $\mathrm{C}-\mathrm{X}$ and $\mathrm{C}-\mathrm{C}$ bond formation protocols (Figure 2b), ${ }^{35-40}$ we questioned whether they can be applied in radical-radical cross coupling with ketyl radicals, leading to a quick access of bulkily substituted alcohols. In complement with the reported radical-radical cross coupling methods which mostly require a hetero atom adjacent to the radical center. In contrast, our interest to find different coupling partners to allylic and benzylic family of compounds, thus enlarge the scope of the synthetic application.

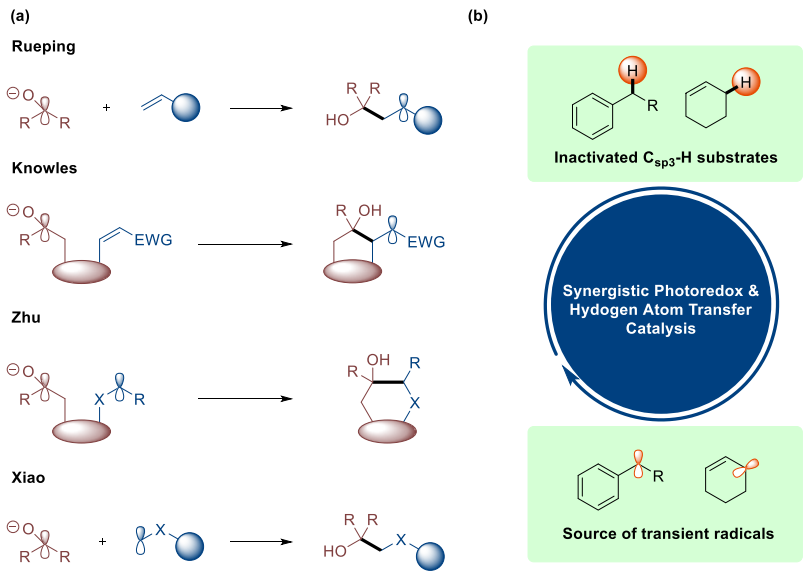

Figure 2. Recent developments in ketyl radical chemistry via photoredox catalysis.

Table 1. Optimization studies

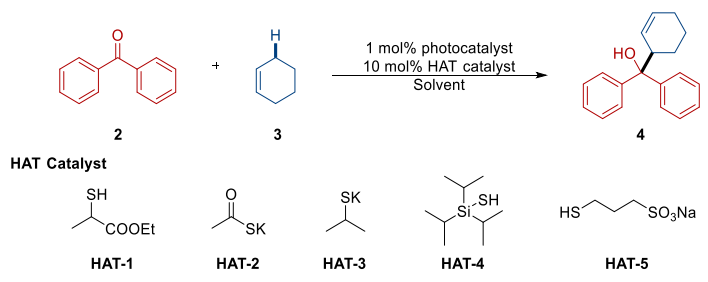

\begin{tabular}{|c|c|c|c|c|}
\hline Entry & $\begin{array}{c}\text { Photocatalyst } \\
\text { (1 } \mathrm{mol} \% \text { ) }\end{array}$ & $\begin{array}{l}\text { HAT cat. } \\
\text { (10 mol\%) }\end{array}$ & $\begin{array}{l}\text { Additives } \\
\text { (10 mol\%) }\end{array}$ & $\begin{array}{c}\text { Re- } \\
\text { sults[a] }\end{array}$ \\
\hline 1 & $\mathrm{Ru}(\mathrm{bpy})_{3}\left(\mathrm{PF}_{6}\right)_{2}$ & HAT-1 & $\mathrm{K}_{2} \mathrm{CO}_{3}$ & N.R. \\
\hline 2 & $\operatorname{Ir}(\text { ppy })_{2}($ bpy $) \mathrm{PF}_{6}$ & HAT-1 & $\mathrm{K}_{2} \mathrm{CO}_{3}$ & N.R. \\
\hline 3 & $\operatorname{Ir}(\text { ppy })_{2}(\mathrm{dtbbpy}) \mathrm{PF}_{6}$ & HAT-1 & $\mathrm{K}_{2} \mathrm{CO}_{3}$ & Trace 4 \\
\hline 4 & $f a c-\operatorname{Ir}(\mathrm{ppy})_{3}$ & HAT-1 & $\mathrm{K}_{2} \mathrm{CO}_{3}$ & $40 \% 4$ \\
\hline $5^{[b]}$ & $f a c-\operatorname{Ir}(\mathrm{ppy})_{3}$ & HAT-1 & $\mathrm{K}_{2} \mathrm{CO}_{3}$ & $58 \% 4$ \\
\hline 6 & $f a c-\operatorname{Ir}(\mathrm{ppy})_{3}$ & HAT-2 & - & $90 \% 4$ \\
\hline 7 & $f a c-\operatorname{Ir}(\mathrm{ppy})_{3}$ & HAT-3 & - & N.D. \\
\hline 8 & $f a c-\operatorname{Ir}(\mathrm{ppy})_{3}$ & HAT-4 & $\mathrm{K}_{2} \mathrm{CO}_{3}$ & $85 \% 4$ \\
\hline 9 & $f a c-\operatorname{Ir}(\mathrm{ppy})_{3}$ & HAT-5 & $\mathrm{K}_{2} \mathrm{CO}_{3}$ & N.D. \\
\hline 10 & - & - & - & N.R. \\
\hline 11 & fac-Ir(ppy) ${ }_{3}$ & - & - & Only 7 \\
\hline $12[c]$ & fac-Ir(ppy $)_{3}$ & HAT-2 & - & N.R. \\
\hline 13 & - & HAT-2 & - & N.R. \\
\hline
\end{tabular}

The reactions were carried out under inert atmosphere $\mathrm{Ar}$ or $\mathrm{N}_{2}$ ) using $0.2 \mathrm{mmol}$ benzophenone and $1.0 \mathrm{mmol}$ cyclohexene in $0.1 \mathrm{M}$ acetone solution. The reaction vials were irradiated by $34 \mathrm{~W}$ Kessil Blue LEDs for $12 \mathrm{hrs}$. [a] Isolated yield. [b] Reaction in DMSO 0.1 M solution. [c] Reaction in the dark. N.R. No reaction. N.D. Not determined, complex mixture obtained.

which exhibits absolute atom economy and be of great interest in green chemistry. ${ }^{41}$ In this article, we report our design of such a reaction and show its potential applications in tertiary alcohol synthesis.

Our model reactions between benzophenone (2) and cyclohexene (3) were examined under various photoredox conditions (Table 1). Photocatalysts that have relatively low reduction potential could not facilitate the transformation (Entry 13 ). The initial use of acid additives (ie. benzoic acid) ${ }^{20-21}$ to fa- 
Table 2. (a) Substrate scope evaluation for various ketones. (b) Substrate scope evaluation for various allylic/benzylic $\mathrm{C}_{\mathrm{sp} 3}-\mathrm{H}$

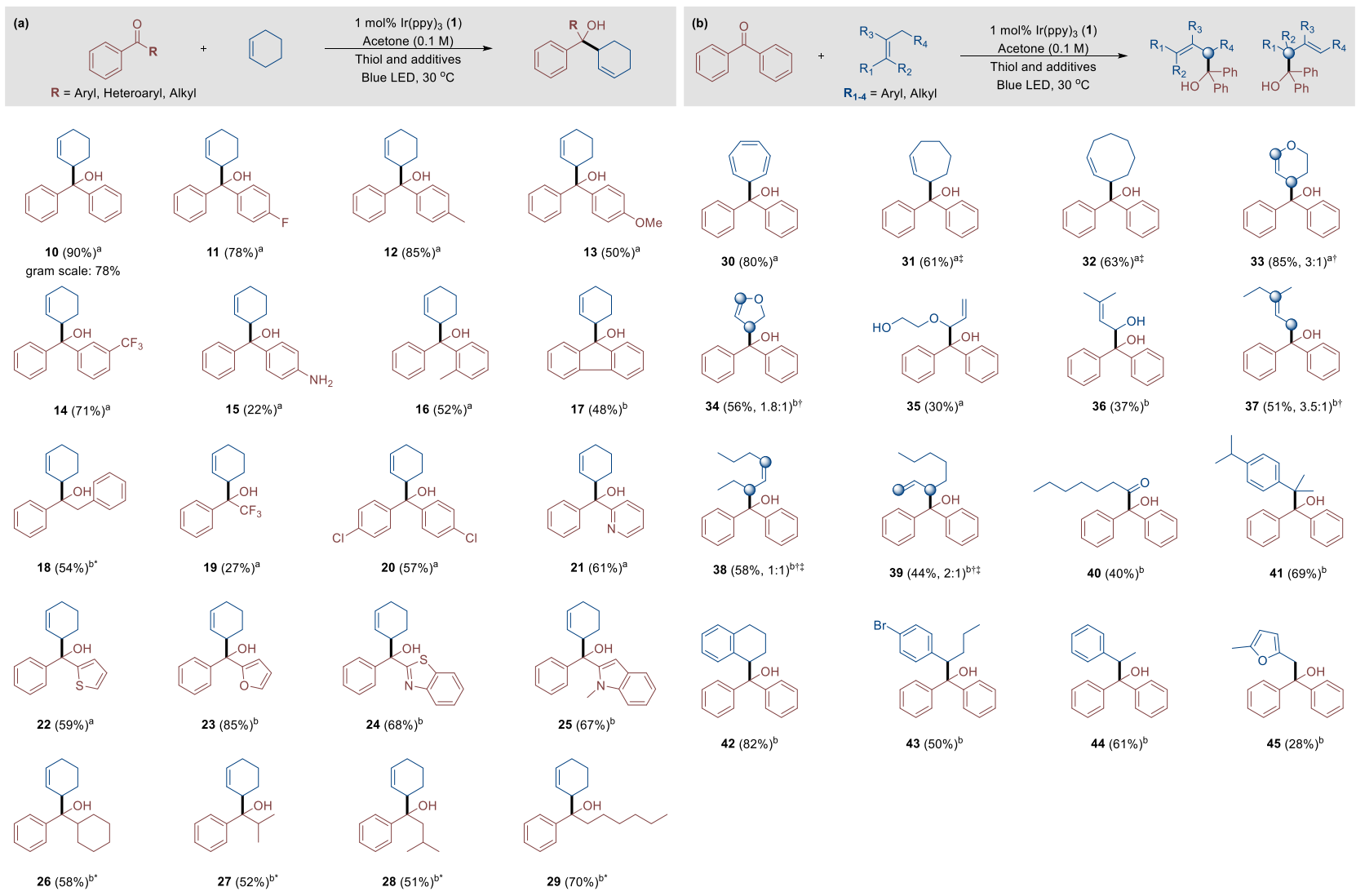

All reactions were carried out under inert atmosphere of Argon, with $0.2 \mathrm{mmol}$ ketone and 1.0 mmol cyclohexene for 12-16 hours (a), or with $0.2 \mathrm{mmol}$ benzophenone and $0.6 \mathrm{mmol}$ olefin/alkyl benzene for 12-16 hours (b) unless otherwise indicated. Yields given in parentheses are isolated yields. a, condition A using KSAc (10 mol\%). b, condition B using tri-isopropylsilanethiol (10 mol\%) and $\mathrm{K}_{2} \mathrm{CO}_{3}(10 \mathrm{~mol} \%)$. Diastereoisomers were obtained without selectivity $(\mathrm{dr} \approx 1: 1)$ for all the cases using unsymmetrical ketones. * Reactions were carried out for 72 hours. $\dagger$ Regioisomers were obtained, the main isomers are depicted in all cases. $\$$ Additional loading of olefin required (see Supplementary Information for more details)

cilitate ketone single electron reduction gave detrimental results while base additives such as carbonate salts increased the reaction efficiency, possibly by accelerating thiolate formation (Entry 4 \& 5). In addition, the reaction works on polar aprotic solvents such as DMSO but the reaction system that contains protic solvents including alcohols or trace water gave rise to a pinacol dimerization product (7). The choice of thiol catalyst is a decisive factor in this protocol. From our screening results (Table 1), we have concluded two optimized conditions, in which, both gave excellent yields of product in the model reaction $(>85 \%$ ). (Entry 6 \& 8).

Condition A features the direct use of inexpensive potassium thioacetate without additional base, while condition $B$ employs tri-isopropylsilanethiol together with potassium carbonate, all in $10 \mathrm{~mol} \%$ loading. Although the triplet state benzophenone has been known as a hydrogen atom transfer catalyst that provides trace coupling product with cyclohexene, our control experiments ruled out this pathway, reaffirming the necessity of all parameters. ${ }^{42}$ (Entry 11-13).

A plausible mechanism for the ketone hydrocarbonation is delineated in figure 3. After initial excitation by blue light
(455 nm), the photosensitizer (1) transforms into its longlived triplet excited-state (lifetime $1900 \mathrm{~ns}$ ). ${ }^{43-44}$ Owing to great reducing ability ( $\mathrm{E}=-1.73 \mathrm{~V}$ vs SCE), ${ }^{45}$ the excited photocatalyst undergoes oxidative quenching by aryl ketone $\left(E_{\text {benzophenone }}=-1.87 \mathrm{~V} \text { vs SCE }\right)^{46-47}$ to generate semi-persistent ketyl radical and higher oxidation state photocatalyst $\left(\mathrm{Ir}^{4+}\right)$ with good oxidizing ability $(\mathrm{E}=+0.77 \mathrm{~V} \text { vs SCE})^{45}$. Consequently, single electron oxidation of in situ generated thiolate gives thiyl radical. We anticipate that the electrophilic thiyl radical can serve as a powerful hydrogen atom transfer catalyst which abstracts the allylic/benzylic $\mathrm{C}_{\mathrm{sp} 3}-\mathrm{H}$ bonds (BDE for cyclohexene $\mathrm{C}-\mathrm{H}_{\text {allylic }}=83.2 \mathrm{kcal} \cdot \mathrm{mol}^{-1}, \mathrm{BDE}$ for toluene $\mathrm{C}$ - $\mathrm{H}_{\text {benzylic }}=89.9 \mathrm{kcal} \cdot \mathrm{mol}^{-1} \mathrm{~J} \cdot{ }^{48-49} \mathrm{In}$ fact, the presence of cyclohexenyl radical 6 was confirmed by the isolation of the TEMPO trapping product $\mathbf{8}$. The transient allylic/benzylic radicals can undergo cross radical-radical coupling with the ketyl radical to afford the desired tertiary alcohols.

With the optimized conditions in hand, we first evaluated the scope of carbonyl alkylation on a wide range of ketones (Table 2a). Diaryl ketones generally provided good coupling efficiency, in spite of steric hindrance. However, ortho-sub- 


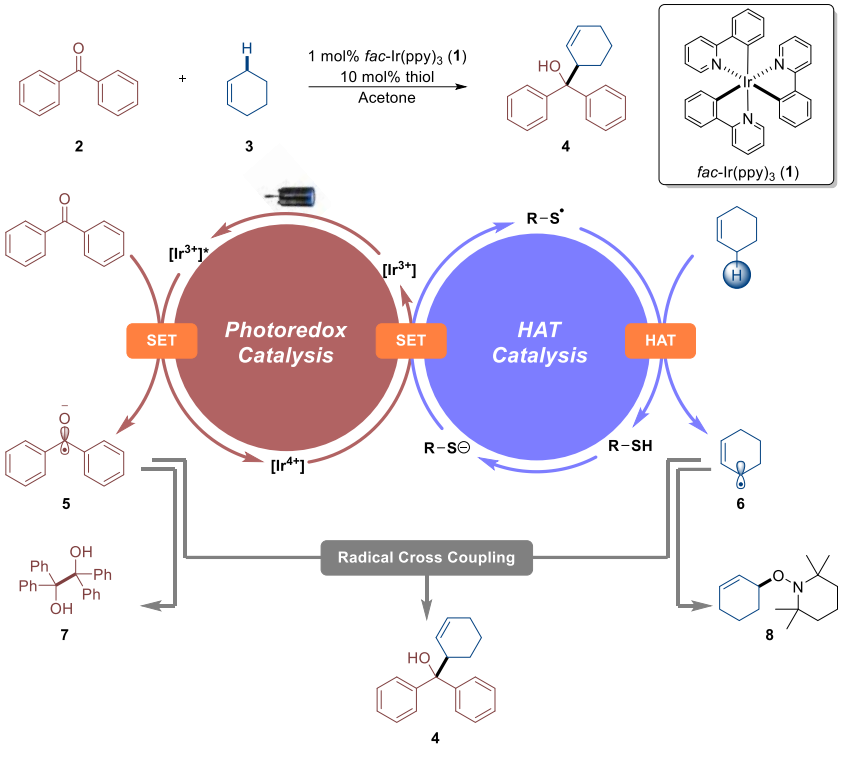

Figure 3. Proposed mechanism for carbonyl alkylation of ketones using photoredox catalysis.

stituted aryl ketone (16) gave significantly lower yield compared with the para- and meta- counterpart (12 and 14). It is interesting that the benzylic $\mathrm{C}_{\mathrm{sp} 3}-\mathrm{H}$ bond on the ketone substrate was not activated by the thiyl radical, hence, the selective cross-coupling with cyclohexene was observed. Strong electron donating substituents such as -OMe (13) or $-\mathrm{NH}_{2}$ (15) present on the ketone substrates imposes negative impact to the reaction outcome. Although these substituents can stabilize the ketyl radical intermediates, they raise the energy barrier for single electron reduction process. The conversion of the ketone substrates in these cases was not completed even after 48 hours. A cyclic diaryl ketone gave a moderate yield of $48 \%$ (17), possibly because of a reduced steric shield which helps to stabilize the ketyl radical intermediate. A gram-scale reaction was performed with the use of minimal amount of catalysts over prolonged reaction times and gave very good yield $(0.1 \mathrm{~mol} \%$ [Ir], 2 mol\% KSAc; 10).

In addition, heterocyclic aryl ketones are viable substrates that produced bulky tertiary alcohols in moderate to good yields (59\%-85\%). Electron deficient heterocycles (pyridine in 21, benzothiazole in 24) and electron enriched heterocycles (furan in $\mathbf{2 3}$, indole in 25 ) did not show any substantial effect in the reactivity. When these non-symmetrical ketones were employed, the alcohol products were obtained in a diastereoisomeric mixture $(\mathrm{dr} \approx 1: 1$ in all the cases).

To our delight, aryl-alkyl ketones can undergo the crossradical coupling reaction efficiently, although extended reaction times are required. This significantly broadens the range of tertiary alcohols that can be synthesized using our benign protocol. Ketones with cyclic alkyl (26) and linear alkyl (29) worked equally well. Moreover, benzylic ketone (18), branched primary and secondary alkyl ketones (27 and 28) also furnished tertiary alcohols with synthetically useful yields (>50\%). Additionally, trifluoro substituted acetophenone reacted, albeit slowly, to form trifluoromethyl tertiary alcohol (19). Even though significant effort has been made to optimize the reaction conditions for the alkylation of dialkyl ketones and aldehydes, no desirable outcome has been achieved so far. It is presumably due to the high reduction potential of the dialkyl ketones and the short lifetime of the aldehyde derived ketyl radicals. The SternVolmer quenching plot shows that the aromatic ketones are good quenchers of the excited state $\left[f a c-\operatorname{Ir}(\mathrm{ppy})_{3}\right]^{*}$, while the aliphatic ketones and alkenes are not (See supplementary information). This explains the limitation of our scope towards the aliphatic ketones.

We next sought to expand this reaction from the model substrate - cyclohexene to other allylic/benzylic systems (Table 2b). Conjugated alkene - cycloheptatriene provided a good yield of $80 \%$ (30), owing to greater stability of the transient radical. Apart from that, cycloalkenes in various ring sizes, both cycloheptene (31) and cis-cyclooctene (32), gave reasonably good results ( $>60 \%)$. Heterocyclic alkenes with a polarized double bond, for example, dihydropyran and dihydrofuran, furnished regioisomeric product mixtures due to the 1,3-allylic rearrangement. Notably, free-hydroxyl containing olefins can be viable substrates for the reaction as well, although a lower yield was observed. It has been challenging to synthesize a carbanion equivalent with such unprotected hydroxyl presenting. Further successful examples with acyclic linear terminal (39), internal olefins (38) and branched olefin (37) reaffirmed the broad applicability of our method, from natural feedstock to latent alkyl carbanion equivalents.

Following our previous success in aldehyde $\mathrm{C}_{\mathrm{sp} 2}-\mathrm{H}$ activation, ${ }^{50}$ we have tested and achieved benzoin condensation type product (40) in the cross-radical coupling between an acyl radical and a ketyl radical. Moreover, a series of benzylic $\mathrm{C}_{\mathrm{sp} 3}-\mathrm{H}$ substrates were successfully employed in the carbonyl alkylation. Tertiary (41) and secondary (both cyclic - 42 and acyclic - 43, 44) benzylic substrates gave moderate to good yields (50-82\%). However, a primary benzylic substrate provided a low yield (45), attributed to the shortlived primary radical intermediate. In addition, it is of considerable value for the tolerance of a bromo substituent, which will allow multi-functionalization of the substrate. It has been problematic for the classical organometallic reagents in this aspect.

Finally, mild reaction condition, a central advantage of this protocol, has been demonstrated in late-stage diversification of advanced, highly functionalized synthetic intermediates and natural products. As illustrated in Table 3, fenofibrate, a top-selling pharmaceutical for treatment of hypercholesterolemia, successfully underwent the cross-radical coupling reaction, forming alcohol 46 in a moderate yield of $64 \%$. The reaction is chemoselective at the carbonyl group, leaving the ester group intact. Furthermore, the potential pharmaceutical precursors (47 and 48) were smoothly synthesized in one step from inexpensive and commercially available substrates.

More beneficially, alkene-containing natural products and derivatives may likewise be employed directly as carbanion equivalent for the carbonyl alkylation. Citronellyl acetate reacted with benzophenone with complete conversion, giving a mixture of regio-isomers, from which, the main isomer (49) was isolated and characterized. Naturally occurring terpenes such as $\alpha$-Pinene and $\gamma$-Terpinene with 
multiple allylic $\mathrm{C}_{\mathrm{sp} 3}-\mathrm{H}$ sites selectively reacted at the ring carbons to deliver the tertiary alcohol products in $53 \%$ and $40 \%$ respectively.

Table 3. Application of visible-light photoredox mediated carbonyl alkylation.

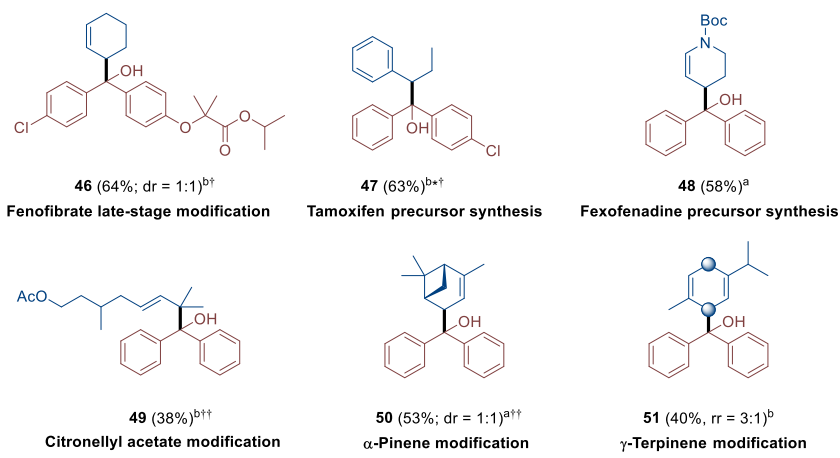

All reactions were carried out under inert atmosphere of Argon, with $0.2 \mathrm{mmol}$ ketone substrate and $0.6 \mathrm{mmol}$ olefin/alkyl benzene for 24 hours, unless otherwise indicated. Yields given in parentheses are isolated yield. a, condition A using KSAc (10 mol\%). b, condition B using tri-isopropylsilanethiol (10 mol\%) and $\mathrm{K}_{2} \mathrm{CO}_{3}(10 \mathrm{~mol} \%)$. * Diastereoisomers were obtained. $\uparrow$ Additional loading of olefin required (see Supplementary Information for more details). $\dagger$ Mixture of regio-isomeric products were obtained; the yield is reported for the main isomer depicted. dr: diastereoisomeric ratio. rr: regioisomeric ratio.

In conclusion, we have developed an efficient protocol for direct hydrocarbonation of aryl ketones to obtain various challenging tertiary alcohols. The reactions proceed via an intermolecular cross radical-radical coupling between a semi-persistent ketyl radical and a transient alkyl radical generated through HAT process using thiol and photoredox catalyst. Our catalytic method eliminates the need of prefunctionalization steps including halogenation and metalation, also avoids the usage of stoichiometric reactive reducing reagents that are harmful to the environment. The quick modification of natural products as well as pharmaceuticals was demonstrated as examples of our mild, simple and effective methodology.

\section{AUTHOR INFORMATION}

\section{Corresponding Author}

*xuewei@ntu.edu.sg

\section{Author Contributions}

$\dagger$ These authors contributed equally.

Notes

The authors declare no competing financial interests.

\section{ASSOCIATED CONTENT}

Supporting Information includes experimental procedures and full characterization of all compounds. This material is available free of charge via the Internet at http://pubs.acs.org.

\section{ACKNOWLEDGMENT}

We gratefully acknowledge National Research Foundation (NRF2016NRF-NSFC002-005) and the Ministry of Education (MOE 2013-T3-1-002), Singapore for financial support of this research.

\section{ABBREVIATIONS}

ppy, 2-phenylpyridine; dtbbpy, 4,4'-Di-tert-butyl-2,2'-dipyridyl; bpy, 2,2'-bipyridyl

\section{REFERENCES}

1. Chen, S.-T.; Fang, J.-M., Preparation of Optically Active Tertiary Alcohols by Enzymatic Methods. Application to the Synthesis of Drugs and Natural Products. J. Org. Chem. 1997, 62, 4349-4357.

2. Li, J. J., Barbier reaction. In Name Reactions: A Collection of Detailed Mechanisms and Synthetic Applications Fifth Edition, Springer International Publishing: Cham, 2014; pp 21-22.

3. Hatano, M.; Ishihara, K., Recent Progress in the Catalytic Synthesis of Tertiary Alcohols from Ketones with Organometallic Reagents. Synthesis 2008, 2008, 1647-1675.

4. $\quad$ Keh, C. C. K.; Wei, C.; Li, C.-J., The Barbier-Grignard-Type Carbonyl Alkylation Using Unactivated Alkyl Halides in Water. J. Am. Chem. Soc. 2003, 125, 4062-4063.

5. Li, C.-J., Organic Reactions in Aqueous Media with a Focus on Carbon-Carbon Bond Formations: A Decade Update. Chem. Rev. 2005, 105, 3095-3166.

6. Zhou, F.; Li, C.-J., The Barbier-Grignard-type arylation of aldehydes using unactivated aryl iodides in water. Nat. Commun 2014, 5, 4254-4260.

7. Toni, M.; Arkaitz, C.; Ruben, M., Metal-Catalyzed Reductive Coupling Reactions of Organic Halides with CarbonylType Compounds. Chem. Eur. J. 2014, 20, 8242-8258.

8. $\quad$ Nguyen, K. D.; Park, B. Y.; Luong, T.; Sato, H.; Garza, V. J.; Krische, M. J., Metal-catalyzed reductive coupling of olefin-derived nucleophiles: Reinventing carbonyl addition. Science 2016, 354, 300-305.

9. Yang, Y.; Perry, I. B.; Lu, G.; Liu, P.; Buchwald, S. L., Copper-catalyzed asymmetric addition of olefin-derived nucleophiles to ketones. Science 2016, 353, 144-150.

10. Wang, H.; Dai, X.-J.; Li, C.-J., Aldehydes as alkyl carbanion equivalents for additions to carbonyl compounds. Nat. Chem. 2016, 9, 374-378.

11. Li, B.-S.; Wang, Y.; Proctor, R. S. J.; Zhang, Y.; Webster, R. D.; Yang, S.; Song, B.; Chi, Y. R., Carbene-catalysed reductive coupling of nitrobenzyl bromides and activated ketones or imines via single-electron-transfer process. Nat. Commun. 2016, 7, 12933-12940.

12. Pitzer, L.; Sandfort, F.; Strieth-Kalthoff, F.; Glorius, F., Intermolecular Radical Addition to Carbonyls Enabled by Visible Light Photoredox Initiated Hole Catalysis. J. Am. Chem. Soc. 2017, 139, 13652-13655.

13. Nicolaou, K. C.; Ellery, S. P.; Chen, J. S., Samarium Diiodide Mediated Reactions in Total Synthesis. Angew. Chem. Int. Ed. 2009, $48,7140-7165$.

14. Krief, A.; Laval, A.-M., Coupling of Organic Halides with Carbonyl Compounds Promoted by SmI2, the Kagan Reagent. Chem. Rev. 1999, 99, 745-778.

15. Molander, G. A.; Harris, C. R., Sequencing Reactions with Samarium(II) Iodide. Chem. Rev. 1996, 96, 307-338.

16. Szostak, M.; Fazakerley, N. J.; Parmar, D.; Procter, D. J., Cross-Coupling Reactions Using Samarium(II) Iodide. Chem. Rev. 2014, 114, 5959-6039.

17. Chciuk, T. V.; Anderson, W. R.; Flowers, R. A., ProtonCoupled Electron Transfer in the Reduction of Carbonyls by Samarium Diiodide-Water Complexes. J. Am. Chem. Soc. 2016, 138, 8738-8741. 
18. Kern, N.; Plesniak, M. P.; McDouall, J. J. W.; Procter, D. J., Enantioselective cyclizations and cyclization cascades of samarium ketyl radicals. Nat. Chem. 2017, 9, 1198-1204.

19. Rono, L. J.; Yayla, H. G.; Wang, D. Y.; Armstrong, M. F.; Knowles, R. R., Enantioselective Photoredox Catalysis Enabled by Proton-Coupled Electron Transfer: Development of an Asymmetric Aza-Pinacol Cyclization. J. Am. Chem. Soc. 2013, 135, 17735-17738.

20. Tarantino, K. T.; Liu, P.; Knowles, R. R., Catalytic KetylOlefin Cyclizations Enabled by Proton-Coupled Electron Transfer. J. Am. Chem. Soc. 2013, 135, 10022-10025.

21. Eleonora, F.; Anthony, M.; Masaki, N.; Sebastian, L.; Magnus, R., Reductive Umpolung of Carbonyl Derivatives with Visible-Light Photoredox Catalysis: Direct Access to Vicinal Diamines and Amino Alcohols via $\alpha$-Amino Radicals and Ketyl Radicals. Angew. Chem. Int. Ed. 2016, 55, 6776-6779.

22. Masaki, N.; Eleonora, F.; Sebastian, L.; Zhen, J.; Magnus, R., Photoredox-Catalyzed Reductive Coupling of Aldehydes, Ketones, and Imines with Visible Light. Angew. Chem. Int. Ed. 2015, 54, 8828-8832.

23. Fava, E.; Nakajima, M.; Nguyen, A. L. P.; Rueping, M., Photoredox-Catalyzed Ketyl-Olefin Coupling for the Synthesis of Substituted Chromanols. J. Org. Chem. 2016, 81, 6959-6964.

24. Lee, K. N.; Ngai, M.-Y., Recent developments in transitionmetal photoredox-catalysed reactions of carbonyl derivatives. Chem. Commun. 2017, 53, 13093-13112.

25. Lee, K. N.; Lei, Z; Ngai, M.-Y., $\beta$-Selective Reductive Coupling of Alkenylpyridines with Aldehydes and Imines via Synergistic Lewis Acid/Photoredox Catalysis. J. Am. Chem. Soc. 2017, 139, 5003-5006.

26. Li, W.; Duan, Y.; Zhang, M.; Cheng, J.; Zhu, C., A photoredox catalyzed radical-radical coupling reaction: facile access to multisubstituted nitrogen heterocycles. Chem. Commun. 2016, 52, 7596-7599.

27. Ding, W.; Lu, L.-Q.; Liu, J.; Liu, D.; Song, H.-T.; Xiao, W.-J., Visible Light Photocatalytic Radical-Radical Cross-Coupling Reactions of Amines and Carbonyls: A Route to 1,2-Amino Alcohols. J. Org. Chem. 2016, 81, 7237-7243.

28. Liu, Y.; Liu, X.; Li, J.; Zhao, X.; Qiao, B.; Jiang, Z., Catalytic enantioselective radical coupling of activated ketones with $\mathrm{N}$-aryl glycines. Chem. Sci. 2018, 9, 8094-8098.

29. Wang, C.; Qin, j.; Shen, X.; Reidel, R.; Harms, K.; Meggers, E., Asymmetric Radical-Radical Cross-Coupling through VisibleLight-Activated Iridium Catalysis. Angew. Chem. Int. Ed. 2016, 55, 685-688.

30. Uraguchi, D.; Kinoshita, N.; Kizu, T.; Ooi, T., Synergic Catalysis of Ionic Bronsted Acid and Photosensitizer for a Redox Neutral Asymmetric $\alpha$-Coupling of $N$-Arylaminomethanes with aldimines. J. Am. Chem. Soc. 2015, 137, 13768-13771.

31. Berger, A. L.; Donabauer, K.; König, B., Photocatalytic Barbier reaction - visible-light induced allylation and benzylation of aldehydes and ketones. Chem. Sci. 2018, 9, 7230-7235.

32. Yi, H.; Zhang, G.; Wang, H.; Huang, Z.; Wang, J.; Singh, A. K.; Lei, A., Recent Advances in Radical C-H Activation/Radical Cross-Coupling. Chem. Rev. 2017, 117, 9016-9085.

33. Davies, H. M. L.; Morton, D., Recent Advances in $\mathrm{C}-\mathrm{H}$ Functionalization. J. Org. Chem. 2016, 81, 343-350.

34. Wencel-Delord, J.; Glorius, F., C-H bond activation enables the rapid construction and late-stage diversification of functional molecules. Nat. Chem. 2013, 5, 369-375.
35. Cuthbertson, J. D.; MacMillan, D. W. C., The direct arylation of allylic $\mathrm{sp}^{3} \mathrm{C}-\mathrm{H}$ bonds via organic and photoredox catalysis. Nature 2015, 519, 74-77.

36. Loh, Y. Y.; Nagao, K.; Hoover, A. J.; Hesk, D.; Rivera, N. R.; Colletti, S. L.; Davies, I. W.; MacMillan, D. W. C., Photoredoxcatalyzed deuteration and tritiation of pharmaceutical compounds. Science 2017, 358, 1182-1187.

37. Jeffrey, J. L.; Petronijević, F. R.; MacMillan, D. W. C., Selective Radical-Radical Cross-Couplings: Design of a Formal $\beta$ Mannich Reaction. J. Am. Chem. Soc. 2015, 137, 8404-8407.

38. Qvortrup, K.; Rankic, D. A.; MacMillan, D. W. C., A General Strategy for Organocatalytic Activation of $\mathrm{C}-\mathrm{H}$ Bonds via Photoredox Catalysis: Direct Arylation of Benzylic Ethers. J. Am. Chem. Soc. 2014, 136, 626-629.

39. Hager, D.; MacMillan, D. W. C., Activation of C-H Bonds via the Merger of Photoredox and Organocatalysis: A Coupling of Benzylic Ethers with Schiff Bases. J. Am. Chem. Soc. 2014, 136, 16986-16989.

40. Petronijević, F. R.; Nappi, M.; MacMillan, D. W. C., Direct $\beta$-Functionalization of Cyclic Ketones with Aryl Ketones via the Marger of Photoredox and Organocatalysis. J. Am. Chem. Soc. 2013, 135, 18323-18326.

41. Trost, B. M., On Inventing Reactions for Atom Economy. Acc. Chem. Res. 2002, 35, 695-705.

42. Fréneau, M.; Hoffmann, N., The Paternò-Büchi reactionMechanisms and application to organic synthesis. J. Photochem. Photobiol. C: Photochem. Rev. 2017, 33, 83-108.

43. Jifu, S.; Wanhua, W.; Jianzhang, Z., Long-Lived RoomTemperature Deep-Red-Emissive Intraligand Triplet Excited State of Naphthalimide in Cyclometalated Ir $^{\text {III }}$ Complexes and its Application in Triplet-Triplet Annihilation-Based Upconversion. Chem. Eur. J. 2012, 18, 8100-8112.

44. Hofbeck, T.; Yersin, H., The Triplet State of $f a c-\operatorname{Ir}(\mathrm{ppy})_{3}$. Inorg. Chem. 2010, 49, 9290-9299.

45. Flamigni, L.; Barbieri, A.; Sabatini, C.; Ventura, B.; Barigelletti, F., Photochemistry and Photophysics of Coordination Compounds: Iridium. In Photochemistry and Photophysics of Coordination Compounds II, Balzani, V.; Campagna, S., Eds. Springer Berlin Heidelberg: Berlin, Heidelberg, 2007; pp 143-203.

46. Wagner, P. J.; Truman, R. J.; Puchalski, A. E.; Wake, R., Extent of charge transfer in the photoreduction of phenyl ketones by alkylbenzenes. J. Am. Chem. Soc. 1986, 108, 7727-7738.

47. Roth, H. G.; Romero, N. A.; Nicewicz, D. A., Experimental and Calculated Electrochemical Potentials of Common Organic Molecules for Applications to Single-Electron Redox Chemistry. Synlett 2016, 27, 714-723.

48. Wu, Y.-D.; Wong, C.-L.; Chan, K. W. K.; Ji, G.-Z.; Jiang, X.-K., Substituent Effects on the $\mathrm{C}-\mathrm{H}$ Bond Dissociation Energy of Toluene. A Density Functional Study. J. Org. Chem. 1996, 61, 746-750.

49. Khursan, S. L.; Mikhailov, D. A.; Yanborisov, V. M.; Borisov, D. I., AM1 calculations of bond dissociation energies. Allylic and benzylic C-H bonds. React. Kinet. Catal. Lett. 1997, 61, 91-95.

50. Vu, M. D.; Das, M.; Liu, X.-W., Direct Aldehyde $\mathrm{C}_{\mathrm{sp} 2}-\mathrm{H}$ Functionalization through Visible-Light-Mediated Photoredox Catalysis. Chem. Eur. J. 2017, 23, 15899-15902. 
Authors are required to submit a graphic entry for the Table of Contents (TOC) that, in conjunction with the manuscript title, should give the reader a representative idea of one of the following: A key structure, reaction, equation, concept, or theorem, etc., that is discussed in the manuscript. Consult the journal's Instructions for Authors for TOC graphic specifications.

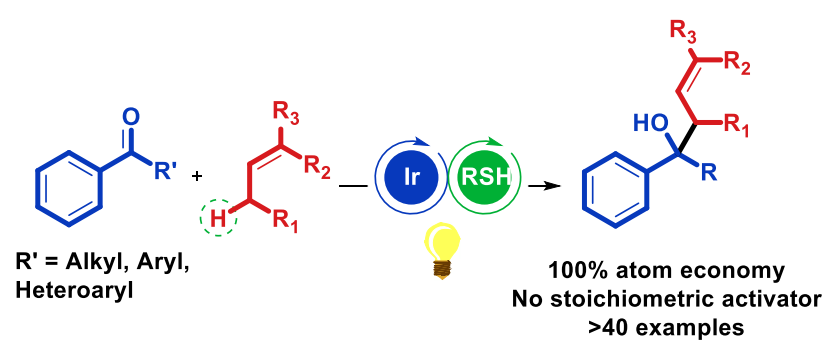

